Epidemiology

\title{
Electronic tools for physical activity and quality of life of medical students during COVID-19 pandemic social isolation
}

\author{
Filipe Silva Barreto $^{1}$ (D), Gabriela Oliveira de Carvalho ${ }^{1}$ (D), Laura Cristina Neves da Silva ${ }^{1}$ (D), \\ Vinicios Ribas dos Santos ${ }^{1}$ (D), Renêe Dominik Carvalho Pereira Osório ${ }^{2}$ (D), \\ Luana Nascimento $^{3}$ (D), Ankilma do Nascimento Andrade Feitosa ${ }^{2}$ (D), \\ Elisangela Vilar de Assis $^{3}$ (D), José Bruno Nunes Ferreira Silva ${ }^{1}$ \\ ${ }^{1}$ Curso de Medicina, Universidade Federal do Tocantins, Palmas, TO, Brasil; ${ }^{2}$ Curso de \\ Medicina, Faculdade Santa Maria, Cajazeiras, PB, Brasil; ${ }^{3}$ Curso de Medicina, Universidade \\ Federal de Campina Grande, Cajazeiras, PB, Brasil
}

Associated Editor: Angelina Zanesco, Departamento de Educação Física, Instituto de Biociências, Universidade Estadual Paulista "Júlio de Mesquita Filho", Rio Claro, SP, Brasil.

\begin{abstract}
Aim: This study investigates the impact of social isolation, weight changes, and physical activity (PA) by the electronic tools (eTools) on the quality of life (QoL) of Brazilian medical students during the COVID-19 pandemic. Methods: In this observational longitudinal study, one hundred eighty-nine medical students completed an e-questionnaire, which used the WHOQOL-Bref instrument to report QoL and sociodemographic characteristics, health conditions, and lifestyles related to PA. Cronbach's alpha and Friedman/Dunn and Spearman's correlation tests were performed. Based on descriptive and inferential statistics, a multiple linear regression was used to evaluate the potential predictors of QoL. Results: The COVID-19 pandemic particularly affected the psychological and social domains. Time spent indoors and a non-ideal BMI had a negative impact on QoL. Being female and sexual minority-group membership were associated with lower QoL scores in the psychological and physical domains, respectively. Access to PA eTools was positively associated with QoL. Being monitored by a physical education teacher contributes to satisfactory PA levels during online classes. Conclusion: This study provides novel insights into the access of PA eTools on QoL during the COVID-19 pandemic. During periods of social distancing, people should pay extra attention to self-care strategies to protect mental health and promote positive lifestyle patterns. In addition, using online PA resources monitored by physical education teachers could be a strategy to maintain satisfactory levels of exercise.
\end{abstract}

Keywords: Covid-19, quality of life, medical students, physical activity, eHealth.

\section{Introduction}

In March 2020, the World Health Organization (WHO) declared that coronavirus disease (COVID-19), caused by the SARS-Cov-2 virus, represented a public health emergency of international concern. Currently, this disease has overburdened health systems worldwide, spreading across the globe in a rising wave. As of January 3, 2021, Brazil ranks first among Latin American countries in the total number of cases and deaths ${ }^{1}$.

Mitigation measures for communities with COVID19 transmission have been established to reduce and delay the spread of the disease. In many countries, including Brazil, people have been instructed to isolate themselves indoors and to maintain social isolation and physical distancing. This non-pharmacological strategy protects individuals, environments, and communities, reducing the number of COVID-19-related deaths ${ }^{2}$. In mid-March, the challenges posed by the pandemic led to the cessation of traditional classrooms in Brazil, as teaching shifted to remote learning throughout the country ${ }^{3}$.

Particularly for medical students, virtual education has caused delays in learning in the clinical environment during this unprecedented time. Students cannot care for patients because there is insufficient personal protective equipment (PPE); in addition, no guidelines have emerged during the COVID-19 crisis $^{4-6}$ to enable students to contribute to clinical experiences. Physical, emotional, and mental illnesses have been reported as a consequence of the current situation ${ }^{6}$.

Long-term social distancing has been shown to decrease quality of life $(\mathrm{QoL})^{7-9}$. It can also make behavioral problems worse, exacerbating weight changes, sedentary patterns, unhealthy diets, and sleep disorders ${ }^{10}$. Social isolation often leads to reduce the physical activity (PA) causing weight gain and obesity. To avoid that due to sedentary behavior, people have been advised to engage in 
PA to maintain their immune function and physical and mental health ${ }^{11}$. The pattern is even more noticeable among medical students, who are known to have worse mental health during periods of social isolation ${ }^{12}$.

Research has shown that young medical students have a lower QoL than young people in general ${ }^{13}$. Gender, having low income, and chronic illnesses, such as stress, anxiety, depression, and obesity, have been argued to be predictors of QoL among Brazilian medical students ${ }^{14,15}$. With the advent of social isolation, the QoL of this population is likely to be disproportionately affected. PA interventions during social distancing in home confinement have been supported as a way of improving long-term health benefits during COVID-19 restrictions ${ }^{16}$. Such interventions have included the use of advanced technology, including electronic tools (eTools) ${ }^{17}$. Throughout this pandemic, however, few studies have investigated the use of eTools for PA during periods of social isolation and home confinement. To date, we know of no study that has analyzed this issue in Brazil. The present study aims to verify the impact of social and physical distancing, weight changes, and eTools on the QoL of medical students during the COVID-19 pandemic.

\section{Methods}

\section{Participants}

The participants were Brazilian medical students, aged 18 or older, from the Federal University of Tocantins in Palmas, and the Federal University of Campina Grande and Santa Maria College, both in Cajazeiras. A convenience sample of 241 students responded to the questionnaire. Of these, 52 questionnaires $(21,57 \%)$ were excluded, due to missing data. The final sample consisted of one hundred eighty-nine students. The study aims were explained to all participants, who voluntarily provided informed consent before the study began. This protocol was approved by the National Research Ethics Commission of the Ministry of Health (CONEP) - C.A.A.E. 33567120.2.1001.5519.

\section{Design}

Data for this longitudinal observational study were collected between August and September 2020. An online questionnaire was administered using Google Forms, with links to the form sent by email, WhatsApp, Facebook, and Instagram.

\section{Measurements}

The participants were asked about their age, sex, smoking habits (yes/no), alcohol consumption (yes/no), sexual orientation (heterosexual and sexual minority: bisexual, homosexual, and others), and marital status. Regarding participants' family income, those who received less than 3 salaries minimum were included in a single group. They were then grouped into three categories, based on age: adolescents (18-19 years), young adults (20-24 years), and adults $(>24 \text { years })^{18}$. They also provided information on their medical histories, with a particular focus on respiratory disease.

The participants reported their body weight and height, which were used to calculate their body mass index (BMI) as body mass $(\mathrm{kg})$ by height squared $\left(\mathrm{m}^{2}\right)$. They were categorized as underweight $\left(<18.5 \mathrm{~kg} / \mathrm{m}^{2}\right)$, normal weight (18.5 to $<25 \mathrm{~kg} / \mathrm{m}^{2}$ ), overweight (25 to $\left.29.9 \mathrm{~kg} / \mathrm{m}^{2}\right)$ or obese $\left(\geq 30 \mathrm{~kg} / \mathrm{m}^{2}\right)^{19}$. In relation to weight change, the participants were asked to choose one of the following options: lost $>3 \mathrm{~kg}$, lost $1-3 \mathrm{~kg}$, maintained weight; gained 1-3 kg; and gained $>3 \mathrm{~kg}$. Accuracy was verified using a scale that ranged from 0 to 10 . The participants also provided information about the amount of time they spent indoors: $8-12 \mathrm{~h} ; 12-16 \mathrm{~h} ; 16-20 \mathrm{~h}$; or 20$24 \mathrm{~h}$ per day ${ }^{10}$. To ascertain their PA-technology use during home confinement, they were asked about YouTube access, fitness/workout applications (apps), and virtual meetings with physical-education teachers.

QoL was evaluated using the World Health Organization's Quality of Life (WHOQOL)-Bref questionnaire, adapted and validated in Brazil ${ }^{20}$. This instrument contains 24 items, measured using a 5-point Likert scale. The items cover four QoL domains: physical health, psychological well-being, social relationships, and environment. The overall QoL and individual domain scores range from 0 to 100 when converted to a linear scale, with higher scores indicating a better perception of QoL.

Participants completed the International Physical Activity Questionnaire Short Form (IPAQ-SF), adapted and validated in Brazil ${ }^{21}$. The questions allowed us to estimate the weekly time spent with different types of physical activities. The scoring protocol was determined based on a previous study, participants were classified into sufficient levels of physical activity (active or very active) and low levels of physical activity (insufficiently active or sedentary $)^{22}$.

\section{Statistical analysis}

The data were analyzed using descriptive statistics. Explanatory variables related to PA practice, including sociodemographic characteristics, health conditions, and lifestyle, were expressed as relative and absolute frequencies. The student's t-test and one-way analysis of variance (ANOVA) were used to analyze the relationships between the explanatory variables and WHOQOL-Bref scores. To explore the association between PA levels and eTools, Chi-square tests were performed. Cronbach's coefficient was calculated to determine internal consistency reliability, with $r \geq 0.7$ considered sufficiently reliable ${ }^{12}$. Spearman's rank correlation and Friedman's/Dunn's tests were performed to analyze the domains ${ }^{23}$. For multiple- 
linear regression models, each domain and overall QoL were included as dependent variables; variables that showed $\mathrm{p}<0.2$ were inserted. For each model, the standardized beta coefficient $(\beta), 95 \%$ confidence interval $(\mathrm{CI})$, and explained variance $\left(\mathrm{R}^{2}\right)$ were calculated. The data were analyzed using SPSS Statistics software version 25 (IBM Corp., Armonk, NY, USA), with a significance level of $\mathrm{p} \leq 0.05$.

\section{Results}

The Cronbach's alpha value for the WHOQOL-Bref questionnaire used in this study was 0.894 . When items in each domain were excluded, the internal consistency coefficient was not significantly reduced. The values achieved were considered sufficiently reliable. A strong correlation was noted between the domains and the whole instrument score. The weakest correlation was found in the environment domain $(\mathrm{p}<0.001, \mathrm{r}=0.667)$, which had the highest mean score $(70.73 \pm 16.25)$, followed by the physical domain $(66.31 \pm 15.72)(\mathrm{p}<0.001)$. The psychological $(59.20 \pm 17.84)$ and social $(61.60 \pm 19.63)$ domains were most affected by social isolation during the COVID-19 pandemic $(\mathrm{p}<0.05)$ (Table 1). The QoL scores of medical students were $64.46 \pm 13.23$, with a median of 65.50 points and scores ranging from 30.75 to 100 .

As shown in Table 2, the sample consisted mainly of single, female, heterosexual participants. Most participants were non-smokers and non-alcohol users without respiratory disease, who had a family income up to three

Table 1 - Descriptive, inferential, and reliability analysis of the WHOQOL-Bref among medical students during social isolation facing COVID-19 pandemic.

\begin{tabular}{lccc}
\hline & Mean \pm SD & Cronbach's alpha & Correlation with WHOQOL-Bref \\
\hline WHOQOL-Bref & $64.46 \pm 13.23$ & $0.894^{\mathrm{a}}$ & \\
D1 physical health & $66.31 \pm 15.72^{\mathrm{d}}$ & $0.871^{\mathrm{b}}$ & $\mathrm{p}<0.001(\mathrm{r}=0.770)^{\mathrm{c}}$ \\
D2 psychological & $59.20 \pm 17.84^{\mathrm{d}}$ & $0.851^{\mathrm{b}}$ & $\mathrm{p}<0.001(\mathrm{r}=0.843)^{\mathrm{c}}$ \\
D3 social relationships & $61.60 \pm 19.63^{\mathrm{d}}$ & $0.889^{\mathrm{b}}$ & $\mathrm{p}<0.001(\mathrm{r}=0.730)^{\mathrm{c}}$ \\
D4 environmental & $70.73 \pm 16.25^{\mathrm{d}}$ & $0.873^{\mathrm{b}}$ & $\mathrm{p}<0.001(\mathrm{r}=0.667)^{\mathrm{c}}$ \\
& $\mathrm{p}<0.001^{\mathrm{d}}$ & & \\
\hline
\end{tabular}

'Cronbach's alpha.

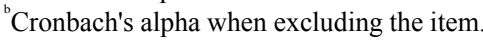

'Spearman's rank correlation with the overall domain (D).

'Friedman's/Dunn's test (D1 vs. D2, p < 0.001; D1 vs. D3, p = 0.016; D1 vs. D4, p=0.132; D2 vs. D3, p = 0.384; D2 vs. D4, p < 0.001; D3 vs. D4, $\mathrm{p}<0.001)$.

Table 2 - Effects of sociodemographic characteristics during social isolation due to COVID-19 on the domain scores for QoL of medical students.

\begin{tabular}{|c|c|c|c|c|c|c|}
\hline Variables & $n(\%)$ & physical & psychological & social & environmental & Overall QoL \\
\hline \multicolumn{7}{|l|}{ Gender } \\
\hline Female & $113(59.8)$ & $64.88 \pm 15.50$ & $56.38 \pm 16.42$ & $62.99 \pm 18.33$ & $70.00 \pm 16.85$ & $63.56 \pm 12.29$ \\
\hline \multirow[t]{2}{*}{ Male } & $76(40.2)$ & $68.43 \pm 15.91$ & $63.38 \pm 19.11$ & $59.53 \pm 21.38$ & $71.82 \pm 15.36$ & $65.79 \pm 14.51$ \\
\hline & & $\mathrm{p}=0.127$ & $p=0.008$ & $\mathrm{p}=0.235$ & $\mathrm{p}=0.453$ & $\mathrm{p}=0.258$ \\
\hline \multicolumn{7}{|l|}{ Age } \\
\hline $18-19$ & 20 (10.6) & $69.15 \pm 17.75$ & $59.75 \pm 21.39$ & $60.25 \pm 18.05$ & $77.05 \pm 9.91$ & $66.55 \pm 13.16$ \\
\hline $20-24$ & $114(60.3)$ & $66.04 \pm 15.74$ & $60.13 \pm 17.68$ & $62.61 \pm 19.20$ & $72.46 \pm 14.42$ & $65.31 \pm 12.59$ \\
\hline \multirow[t]{2}{*}{$>24$} & $55(29.1)$ & $65.84 \pm 15.11$ & $57.05 \pm 16.92$ & $60.00 \pm 21.22$ & $64.84 \pm 18.20$ & $61.93 \pm 14.40$ \\
\hline & & $\mathrm{p}=0.694$ & $\mathrm{p}=0.572$ & $\mathrm{p}=0.686$ & $\mathrm{p}=0.003$ & $\mathrm{p}=0.227$ \\
\hline \multicolumn{7}{|l|}{ Sexual orientation } \\
\hline Heterosexual & $158(83.6)$ & $67.69 \pm 14.76$ & $60.18 \pm 17.39$ & $62.13 \pm 20.04$ & $70.94 \pm 16.68$ & $65.23 \pm 13.07$ \\
\hline \multirow[t]{2}{*}{ Sexual minority } & $31(16.4)$ & $59.26 \pm 18.66$ & $54.16 \pm 19.49$ & $58.90 \pm 17.47$ & $69.68 \pm 14.10$ & $60.50 \pm 13.57$ \\
\hline & & $\mathrm{p}=0.023$ & $\mathrm{p}=0.086$ & $\mathrm{p}=0.405$ & $\mathrm{p}=0.694$ & $\mathrm{p}=0.068$ \\
\hline \multicolumn{7}{|l|}{ Single } \\
\hline No & $13(6.9)$ & $64.54 \pm 17.06$ & $56.38 \pm 18.61$ & $56.23 \pm 22.40$ & $57.31 \pm 17.49$ & $58.62 \pm 16.21$ \\
\hline \multirow[t]{2}{*}{ Yes } & $176(93.1)$ & $66.44 \pm 15.66$ & $59.40 \pm 17.82$ & $61.99 \pm 19.42$ & $71.72 \pm 15.76$ & $64.89 \pm 12.94$ \\
\hline & & $\mathrm{p}=0.675$ & $\mathrm{p}=0.557$ & $\mathrm{p}=0.308$ & $\mathrm{p}=0.002$ & $\mathrm{p}=0.099$ \\
\hline
\end{tabular}


Table 2 - continued

\begin{tabular}{|c|c|c|c|c|c|c|}
\hline Variables & n(\%) & physical & psychological & social & environmental & Overall QoL \\
\hline \multicolumn{7}{|c|}{ Family income } \\
\hline Up to 3 & $76(40.2)$ & $66.34 \pm 15.12$ & $59.88 \pm 17.68$ & $62.58 \pm 19.50$ & $64.26 \pm 13.73$ & $63.27 \pm 13.03$ \\
\hline \multirow[t]{2}{*}{$>3$} & $113(59.8)$ & $66.28 \pm 16.18$ & $58.73 \pm 18.00$ & $60.94 \pm 19.77$ & $75.08 \pm 16.43$ & $65.26 \pm 13.37$ \\
\hline & & $\mathrm{p}=0.980$ & $p=0.666$ & $\mathrm{p}=0.574$ & $\mathrm{p}<0.001$ & $\mathrm{p}=0.311$ \\
\hline \multicolumn{7}{|c|}{ Smoking habit } \\
\hline No & $181(95.8)$ & $66.85 \pm 15.40$ & $59.50 \pm 17.81$ & $61.56 \pm 19.67$ & $70.96 \pm 16.11$ & $64.71 \pm 13.23$ \\
\hline \multirow[t]{2}{*}{ Yes } & $8(4.2)$ & $54.13 \pm 19.11$ & $52.38 \pm 18.12$ & $62.50 \pm 19.88$ & $65.63 \pm 19.86$ & $58.66 \pm 12.67$ \\
\hline & & $\mathrm{p}=0.025$ & $\mathrm{p}=0.270$ & $\mathrm{p}=0.895$ & $\mathrm{p}=0.365$ & $\mathrm{p}=0.311$ \\
\hline \multicolumn{7}{|c|}{ Alcohol consumption } \\
\hline No & $114(60.3)$ & $68.32 \pm 15.26$ & $61.39 \pm 18.24$ & $63.93 \pm 19.40$ & $71.53 \pm 16.49$ & $66.29 \pm 13.16$ \\
\hline \multirow[t]{2}{*}{ Yes } & $75(39.7)$ & $63.24 \pm 16.01$ & $55.87 \pm 16.78$ & $58.05 \pm 19.58$ & $69.52 \pm 15.92$ & $61.67 \pm 12.94$ \\
\hline & & $\mathrm{p}=0.029$ & $\mathrm{p}=0.037$ & $\mathrm{p}=0.044$ & $\mathrm{p}=0.408$ & $\mathrm{p}=0.018$ \\
\hline \multicolumn{7}{|c|}{ Duration of time spent indoors } \\
\hline $8-12 \mathrm{~h}$ & $19(10.1)$ & $77.53 \pm 11.90$ & $68.79 \pm 13.89$ & $69.05 \pm 18.20$ & $74.79 \pm 14.67$ & $72.54 \pm 11.81$ \\
\hline $12-16 \mathrm{~h}$ & $25(13.2)$ & $66.28 \pm 13.26$ & $60.80 \pm 17.63$ & $61.24 \pm 19.98$ & $67.96 \pm 16.52$ & $64.07 \pm 13.21$ \\
\hline $16-20 \mathrm{~h}$ & $50(26.4)$ & $65.90 \pm 17.30$ & $60.56 \pm 17.69$ & $59.60 \pm 19.19$ & $70.40 \pm 18.47$ & $64.12 \pm 14.59$ \\
\hline \multirow[t]{2}{*}{$20-24 \mathrm{~h}$} & $95(50.3)$ & $64.28 \pm 15.39$ & $56.14 \pm 18.09$ & $61.25 \pm 19.99$ & $70.82 \pm 15.30$ & $63.12 \pm 12.37$ \\
\hline & & $p=0.009$ & $\mathrm{p}=0.031$ & $\mathrm{p}=0.350$ & $\mathrm{p}=0.589$ & $p=0.043$ \\
\hline
\end{tabular}

times the Brazilian minimum wage. The amount of time spent indoors during social isolation ranged from 8-12 h (10.1\%) to $20-24 \mathrm{~h}(50.3 \%)$. Each domain score was compared with the variables in Table 2. In the physical domain, heterosexual, non-smoking medical students and those who reported spending fewer hours at home had higher QoL scores. In the psychological domain, participants who were female and spent more time indoors had lower QoL scores. Similarly, individuals who reported using alcohol had statistically lower scores in the physical, psychological, and social domains than non-alcohol users. Participants who were single and under 24 years old, earning more than 3 times the minimum wage, scored higher in the environmental domain.

Table 3 presents the domain-score differences for participants in the groups for weight-change, BMI, and PA-eTool use. The participants had an average age of $23.4 \pm 4.2$ (range: $18-48$ years) and an average BMI of $23.58 \pm 3.61$ (range: 17.16-35.44). Seven participants were underweight, 37 were overweight, and 13 were

Table 3 - Effects of health conditions, weight change, and eTools accessed during social isolation due to COVID-19 on the domain scores for QoL of medical students.

\begin{tabular}{|c|c|c|c|c|c|c|}
\hline Variables & $\mathbf{n}(\%)$ & physical & psychological & social & environmental & Overall QoL \\
\hline \multicolumn{7}{|c|}{ Respiratory disease } \\
\hline No & $152(80.4)$ & $66.82 \pm 15.94$ & $60.09 \pm 17.56$ & $62.32 \pm 19.18$ & $70.57 \pm 16.67$ & $64.95 \pm 13.58$ \\
\hline \multirow[t]{2}{*}{ Yes } & $37(19.6)$ & $64.19 \pm 14.82$ & $55.54 \pm 18.74$ & $58.62 \pm 21.39$ & $71.41 \pm 14.59$ & $62.44 \pm 11.67$ \\
\hline & & $\mathrm{p}=0.362$ & $\mathrm{p}=0.165$ & $\mathrm{p}=0.305$ & $\mathrm{p}=0.779$ & $\mathrm{p}=0.302$ \\
\hline \multicolumn{7}{|c|}{ Weight change } \\
\hline Decreased & $52(27.5)$ & $66.25 \pm 17.37$ & $59.94 \pm 16.78$ & $60.12 \pm 19.47$ & $71.38 \pm 15.07$ & $64.42 \pm 12.20$ \\
\hline Stable & $50(26.5)$ & $68.40 \pm 15.90$ & $61.46 \pm 18.68$ & $63.74 \pm 19.90$ & $69.78 \pm 17.01$ & $65.85 \pm 14.74$ \\
\hline \multirow[t]{2}{*}{ Increased } & $87(46.0)$ & $65.14 \pm 14.61$ & $57.45 \pm 17.98$ & $61.25 \pm 19.70$ & $70.89 \pm 16.65$ & $63.68 \pm 13.00$ \\
\hline & & $\mathrm{p}=0.507$ & $\mathrm{p}=0.423$ & $\mathrm{p}=0.634$ & $\mathrm{p}=0.878$ & $\mathrm{p}=0.656$ \\
\hline \multicolumn{7}{|l|}{ BMI } \\
\hline Non-ideal & $57(30.2)$ & $64.39 \pm 15.30$ & $54.02 \pm 15.57$ & $56.51 \pm 17.43$ & $65.77 \pm 16.32$ & $60.17 \pm 12.26$ \\
\hline \multirow[t]{2}{*}{ Normal } & $132(69.8)$ & $67.14 \pm 15.89$ & $61.43 \pm 18.34$ & $63.80 \pm 20.18$ & $72.87 \pm 15.81$ & $66.31 \pm 13.25$ \\
\hline & & $\mathrm{p}=0.271$ & $\mathrm{p}=0.005$ & $\mathrm{p}=0.019$ & $\mathrm{p}=0.006$ & $\mathrm{p}=0.003$ \\
\hline
\end{tabular}


Table 3 - continued

\begin{tabular}{|c|c|c|c|c|c|c|}
\hline Variables & n(\%) & physical & psychological & social & environmental & Overall QoL \\
\hline \multicolumn{7}{|c|}{ eTools for practicing physical activity } \\
\hline \multicolumn{7}{|l|}{ Youtube } \\
\hline No & $120(63.5)$ & $65.65 \pm 16.18$ & $57.93 \pm 18.40$ & $59.56 \pm 20.64$ & $69.91 \pm 17.29$ & $63.26 \pm 13.35$ \\
\hline \multirow[t]{2}{*}{ Yes } & $69(36.5)$ & $67.45 \pm 14.95$ & $61.39 \pm 16.72$ & $65.14 \pm 17.32$ & $72.16 \pm 14.29$ & $66.54 \pm 12.87$ \\
\hline & & $\mathrm{p}=0.450$ & $\mathrm{p}=0.200$ & $\mathrm{p}=0.059$ & $\mathrm{p}=0.361$ & $\mathrm{p}=0.102$ \\
\hline \multicolumn{7}{|l|}{ Apps } \\
\hline No & $123(65.1)$ & $63.81 \pm 15.42$ & $56.95 \pm 18.25$ & $59.18 \pm 19.53$ & $68.80 \pm 16.27$ & $62.19 \pm 12.76$ \\
\hline \multirow[t]{2}{*}{ Yes } & $66(34.9)$ & $70.95 \pm 15.33$ & $63.38 \pm 16.37$ & $66.11 \pm 19.16$ & $74.32 \pm 15.72$ & $68.69 \pm 13.16$ \\
\hline & & $\mathrm{p}=0.003$ & $\mathrm{p}=0.018$ & $\mathrm{p}=0.020$ & $\mathrm{p}=0.026$ & $\mathrm{p}=0.001$ \\
\hline \multicolumn{7}{|c|}{ Virtual meetings } \\
\hline No & $172(91.0)$ & $65.83 \pm 15.28$ & $58.76 \pm 17.66$ & $60.78 \pm 19.76$ & $69.79 \pm 16.36$ & $63.79 \pm 13.22$ \\
\hline \multirow[t]{2}{*}{ Yes } & $17(9.0)$ & $71.12 \pm 19.59$ & $63.65 \pm 19.51$ & $69.82 \pm 16.67$ & $80.24 \pm 11.80$ & $71.21 \pm 11.70$ \\
\hline & & $\mathrm{p}=0.187$ & $\mathrm{p}=0.282$ & $\mathrm{p}=0.070$ & $\mathrm{p}=0.011$ & $\mathrm{p}=0.027$ \\
\hline
\end{tabular}

obese; they were analyzed together as a single group. Weight gain and loss were reported by $46 \%$ and $27.5 \%$ of participants, respectively. Most participants used eTools $(\mathrm{n}=102 ; 54 \%)$ as a source of PA information, preferring YouTube and digital apps. Participants with normal BMI who used technology had higher psychological, social, and environmental scores than those who did not. Gaining or losing weight, using the YouTube platform, or those who suffered from respiratory diseases were factors that did not affect domain scores. However, participants who used digital apps for PA presented higher QoL values for all domains than those who did not. Having virtual meetings with physical education teachers contributed to higher scores in the environmental domain (Table 3).

Regarding overall QoL, alcohol users, participants with BMI changes, and those who spent more than $12 \mathrm{~h}$ indoors presented lower scores. In addition, access to workout/fitness apps and virtual meetings with physicaleducation teachers were significantly associated with higher QoL means (Table 2 and 3).

Table 4 presents the results of the multiple linear regression analysis. In the physical domain, sexual orien-

Table 4 - Multiple linear regression analysis for WHOQOL-Bref domains during social isolation due to COVID-19.

\begin{tabular}{|c|c|c|c|c|c|}
\hline Variable & Physical & Psychological & Social & Environmental & Overall QoL \\
\hline & $\beta[95 \% \mathrm{CI}]$ & $\beta[95 \% \mathrm{CI}]$ & $\beta[95 \% \mathrm{CI}]$ & $\beta[95 \% \mathrm{CI}]$ & $\beta[95 \% \mathrm{CI}]$ \\
\hline \multirow[t]{2}{*}{ Gender } & -0.099 & -0.198 & & ---- & \\
\hline & {$[-7.70,1.34]$} & {$[-12.31,-2.05]^{* *}$} & & & \\
\hline \multirow[t]{2}{*}{ Age } & & ---- & & -0.152 & ---- \\
\hline & & & & {$[-7.75,-0.42]^{*}$} & \\
\hline \multirow[t]{2}{*}{ Sexual orientation } & -0.178 & -0.090 & & & -0.098 \\
\hline & {$[-13.37,-1.72]^{*}$} & {$[-10.91,2.25]$} & & & {$[-8.37,1.38]$} \\
\hline \multirow[t]{2}{*}{ Single } & & ---- & & 0.153 & 0.103 \\
\hline & & & & {$[1.12,18.46]^{*}$} & {$[-1.76,12.52]$} \\
\hline \multirow[t]{2}{*}{ Family income } & & ---- & & 0.295 & ---- \\
\hline & & & & {$[5.46,14.05]^{* * *}$} & \\
\hline \multirow[t]{2}{*}{ Smoking } & -0.151 & & & & \\
\hline & {$[-22.48,-0.96]^{*}$} & & & & \\
\hline \multirow[t]{2}{*}{ Alcohol } & -0.095 & -0.134 & -0.128 & ---- & -0.142 \\
\hline & {$[-7.48,1.38]$} & {$[-9.77,0.06]$} & {$[-10.74,0.46]$} & & {$[-7.47,-0.20]^{*}$} \\
\hline \multirow[t]{2}{*}{ Respiratory disease } & ---- & -0.087 & & ---- & \\
\hline & & {$[-9.89,2.09]$} & & & \\
\hline Duration of time spent indoors & -0.0183 & -0.165 & & & -0.184 \\
\hline
\end{tabular}


Table 4 - continued

\begin{tabular}{|c|c|c|c|c|c|}
\hline Variable & Physical & Psychological & Social & Environmental & Overall QoL \\
\hline & {$[-5.07,-0.64]^{*}$} & {$[-5.42,-0.41]^{*}$} & & & {$[-4.19,-0.63]^{* *}$} \\
\hline \multirow[t]{2}{*}{ BMI } & ---- & -0.190 & -0.158 & -0.102 & -0.185 \\
\hline & & {$[-12.61,-2.14]^{* *}$} & {$[-12.71,-0.76]^{*}$} & {$[-8.30,1.09]$} & {$[-9.19,-1.44]^{* *}$} \\
\hline \multirow[t]{2}{*}{ Youtube } & & & 0.081 & ---- & -0.009 \\
\hline & & & {$[-2.80,9.41]$} & & {$[-4.21,3.72]$} \\
\hline \multirow[t]{2}{*}{ Apps } & 0.196 & 0.163 & 0.098 & 0.112 & 0.189 \\
\hline & {$[1.99,10.91]^{* *}$} & {$[1.07,11.12]^{*}$} & {$[-2.19,10.26]$} & {$[-0.64,8.24]$} & {$[1.21,9.27]^{*}$} \\
\hline \multirow[t]{2}{*}{ Virtual Meetings } & 0.103 & ---- & 0.114 & 0.158 & 0.151 \\
\hline & {$[-1.83,13.10]$} & & {$[-1.86,17.41]$} & {$[1.60,16.32]^{*}$} & {$[0.74 ; 13.18]^{*}$} \\
\hline $\mathrm{R}^{2}$ & $17.7 \%$ & $17.7 \%$ & $8.9 \%$ & $23.6 \%$ & $18.8 \%$ \\
\hline
\end{tabular}

tation $(\beta=-0.17 ; \mathrm{p}=0.011)$ and smoking habits $(\beta=-0.15 ; p=0.033)$ were important predictors of this model. Being female $(\beta=-0.19 ; \mathrm{p}=0.006)$ was found to be negatively associated with the psychological domain. Only the alcohol consumption variable remained associated with overall QoL $(\beta=-0.14 ; p=0.039)$. Spending more than $12 \mathrm{~h}$ indoors was negatively associated with overall QoL and both the physical and psychological domains. Similarly, participants with non-ideal BMI indices had more negative perceptions of their overall QoL and psychological and social domains. The important predictors in the environmental domain were age $(\beta=-0.52$; $\mathrm{p}=0.029)$, being single $(\beta=0.15 ; \mathrm{p}=0.027)$ and family income $(\beta=0.29 ; \mathrm{p}<0.001)$. Using eTools was significantly and positively associated with overall QoL and the physical, psychological, and environmental domains $(\mathrm{p}<0.05)$.

The relationship between the use of eTools and PA levels is described in Table 5. In this study, 119 (63\%) participants showed satisfactory levels of PA. A greater proportion of participants who had accessed eTools exhib-

Table 5 - Association between accessing the eTools and physical activity levels of medical students during isolation due to COVID-19.

\begin{tabular}{lccc}
\hline Variables & Sufficient level of PA & Low level of PA & p \\
\hline eTools & $47(54)$ & $40(46)$ & 0.019 \\
No & $72(70.6)$ & $30(29.4)$ & \\
Yes & & & \\
Youtube & $73(60.8)$ & $47(39.2)$ & 0.424 \\
No & $46(66.7)$ & $23(33.3)$ & \\
Yes & & & \\
Apps & $72(58.5)$ & $51(41.5)$ & 0.085 \\
No & $47(71.2)$ & $19(28.8)$ & \\
Yes & & & \\
Virtual meetings & $104(60.5)$ & $68(39.5)$ & 0.024 \\
No & $15(88.2)$ & $2(11.8)$ & \\
Yes & & & \\
\hline
\end{tabular}

ited satisfactory levels of PA, compared to those who had not used technology $(70.6 \%$ vs. $54 \%$; $p=0.019)$. This phenomenon was statistically significant for participants who had performed physical activity through virtual meetings with a physical activity teacher $(82.5 \%$ vs. $60.5 \%$; $\mathrm{p}=0.024)$ (Table 5).

\section{Discussion}

This study has analyzed the influence of sociodemographic factors and PA-eTool access on the QoL of medical students during the period of social confinement caused by the COVID-19 pandemic. Consistent with our hypotheses, spending more time at home affects wellbeing and has immediate negative consequences for mental and physical health. The results also suggest an association between BMI and QoL impairment. However, eTool interventions are positively associated with more positive perceptions of the physical, psychological, and environmental domains, as well as overall scores.

When it comes to social distancing during the COVID-19 pandemic, a global study has shown that life satisfaction can be mitigated, affecting psychological well-being ${ }^{9}$. In line with earlier research, this study has adopted a pioneering approach in Brazil, arguing that social distancing has overwhelmed the QoL of medical students. Undergraduate medical students have presented anxiety and depressive symptoms while confined at home during this pandemic ${ }^{24,25}$. The measures taken by colleges and universities to prevent students from getting or spreading the virus - including replacing traditional classroom teaching with emergency remote education without physical contact; cancelling classes around the country; and failing to develop strategies to maintain clinical practice safely as an aspect of student training - are challenges that contribute to mental illness, alongside additional stress $^{12,26,27}$.

Our findings highlight the negative impact that the pandemic has had on the mental health of female students, 
which is consistent with previous research on COVID-19 and medical students ${ }^{12,25}$. A Brazilian meta-analysis has shown that female medical students have lower QoL scores in the psychological domain than male students ${ }^{14}$. These results underline the importance of creating mechanisms that can provide effective psychological support to students in social- and physical-distancing situations. eHealth tools, such as telemedicine, can lead to a new way of caring for people by putting mental health first.

Our results also indicate that students who identify with sexual minority groups have a propensity toward lower means in the physical domain. Lesbian, gay, and bisexual individuals who live in such negative environments face high stressor rates, associated with physical illness, which can be more severe in this group than among heterosexual individuals ${ }^{28}$. Age, higher family income, and being single were factors associated with better QoL in the environmental domain. Alcohol users perceived their QoL more negatively. Further studies are needed to explore the impact of these variables on the QoL of medical students confined to their homes during the pandemic crisis.

Interestingly, in the present study, maintaining a stable weight did not influence QoL during social distancing. The evidence revealed that a short-term lifestyle event related to weight-change self-perception did not impact the QoL of medical students. In our sample, almost all students who were classified as overweight/obese gained weight $(\mathrm{n}=30 ; 60 \%)$ or lost weight $(\mathrm{n}=17 ; 34 \%)$ $(\mathrm{p}=0.001)$. Of the underweight participants, only two maintained the same weight $(\mathrm{p}=0.001)$.

Having a higher BMI is a risk factor for poor outcome in COVID-19 patients ${ }^{29,30}$. Our data confirm those of Serinolli and Novaretti ${ }^{31}$, who found that obese medical students had a lower QoL than non-obese students, specifically in the physical and psychological domains. The present study also shows that underweight students have lower QoL scores, both overall and in the psychological domain. College students who have previously experienced a healthy lifestyle tend to pay more attention to their diet and exercise during lockdown ${ }^{32}$. Although this study has not investigated dietary patterns, efforts to improve an unhealthy diet during social isolation can contribute to BMI-index changes. It is clear that eHealth-education interventions that promote accurate weight management are needed during pandemic periods.

Considering the impact of technology on society during the COVID-19 pandemic, staying connected while staying physically removed is a good strategy for maintaining favorable levels of $\mathrm{QoL}^{9}$. We and others recommend the use of digital platforms during the current health crisis to promote the adoption of PA practice and motivate students to maintain it. In addition, students should maintain health measures and seek alternative strategies, such as home-based PA, which can include social-media and video-search sites, such as YouTube ${ }^{33,34}$. In addition, webbased video-conferencing platforms can allow physicaleducation teachers to monitor exercises ${ }^{35}$. As the present study indicates, physical-education teachers are valuable during online classes, in that their instruction is linked to the satisfactory PA levels and improvement of QoL. The motivation and evaluation provided by physical-education teachers using remote-communication channels deserve further attention during this pandemic time $\mathrm{e}^{36,37}$. Although YouTube is one of the most popular social media platforms in the world, no impact on QoL was found among medical students. This platform allows users to generate and access general content, which is not specifically focused on PA. However, workout/fitness apps play an important role in providing feedback and allowing users to self-monitor calories burned, distance, heart rate, and weight status.

Overall, PA is a strategy for self-care. Practicing it with support can help people maintain proper health and physical function during a pandemic ${ }^{16,38}$. These findings also highlight the important role that universities can and must play by promoting eTools and new strategies that emphasize PA, in order to prevent student mental-health problems and to open up new pathways toward improving QoL during periods of social isolation.

\section{Limitations}

This study has some limitations. First, it has focused on a specific group of undergraduate students at three Brazilian universities. However, despite this limitation, the results are similar to those of other studies that have investigated medical students in home confinement during the COVID-19 pandemic ${ }^{12,24}$. Because the data were selfreported, there was a risk that weight-change status could a weak variable; to counteract this possibility, the accuracy of answers was measured using a numerical rating scale (1-10) which averaged 8.6, a finding very similar to Zachary et al. ${ }^{10}$ The present cross-sectional study has limited causal inference. To explore long-term consequences, this research must be expanded to explore the impact of relaxing social-distancing guidelines. In addition, the present study has deliberately focused on QoL and eTools used for PA. Future research could examine levels of regular PA practice associated with eTools and QoL. Limitations aside, this is the first Brazilian study to present evidence of QoL impairment among medical students, showing that PA eTools play a particularly important role during the present pandemic crisis.

\section{Conclusion}

Medical students' quality of life has been impacted by social isolation due to the COVID-19 pandemic. To 
mitigate this scenario, people forced to spend much of their time indoors can use digital platforms as a PA tool and additionally be monitored by physical education teachers, to help them maintain positive lifestyle patterns. We recommend incorporating eHealth support, specifically focused on mental health and well-being, during this and future pandemic crises.

\section{Conflict of interest}

The authors declare that they have no conflict of interest.

\section{Acknowledgments}

The authors would like to thank UFT and CNPq for the support given to the development of this research work.

\section{References}

1. World Health Organization. Coronavirus disease 2019 (COVID-19) Weekly Epidemiological Update. World Health Organization; 2021. https://www.who.int/publications/m/item/weekly-epidemiological-update-5-january2021

2. Valenti VE, Menezes PL, de Abreus Vieira GNA, Garner DM. Social distancing measures could have reduced estimated deaths related to COVID-19 in Brazil. J Hum Growth Dev. 2020;30:164-9. doi:10.7322/jhgd.v30.10360

3. Silva LLSD, Lima AFR, Polli DA, Razia PFS, Pavão LFA, et al. Social distancing measures in the fight against COVID-19 in Brazil: description and epidemiological analysis by state. Cad Saude Publica. 2020;36:e0185020. doi:10.1590/0102-311X00185020

4. Rose S. Medical Student Education in the Time of COVID-19. JAMA. 2020;323:2131-2. doi:10.1001/jama.2020.5227

5. Ahmed H, Allaf M, Elghazaly H. COVID-19, and medical education. Lancet Infect Dis. 2020;20:777-8. doi:10.1016/ S1473-3099(20)30226-7

6. Chandratre S. Medical Students and COVID-19: Challenges and Supportive Strategies. J Med Educ Curric Dev. 2020;7:2382120520935059. doi:10.1177/ 2382120520935059

7. Omary MB, Eswaraka J, Kimball SD, Moghe PV, Panettieri RA Jr, Scotto KW. The COVID-19 pandemic and research shutdown: staying safe and productive. J Clin Invest. 2020;130:2745-8. doi:10.1172/JCI138646

8. Brooks SK, Webster RK, Smith LE, Woodland L, Wessely S, Greenberg N, et al. The Psychological Impact of Quarantine and How to Reduce It: Rapid Review of the Evidence. The Lancet. 2020;395:912-20. doi:10.1016/S0140-6736 (20)30460-8

9. Ammar A, Chtourou H, Boukhris O, Trabelsi K, Masmoudi L, Brach M, et al. COVID-19 Home Confinement Negatively Impacts Social Participation and Life Satisfaction: A Worldwide Multicenter Study. Int J Environ Res Public Health. 2020;17:6237. doi:10.3390/ijerph17176237

10. Zachary Z, Brianna F, Brianna L, Garrett P, Jade W, Alyssa $\mathrm{D}$, et al. Self-quarantine and weight gain related risk factors during the COVID-19 pandemic. Obes Res Clin Pract. 2020;14:210-16. doi:10.1016/j.orcp.2020.05.004

11. Chen P, Mao L, Nassis GP, Harmer P, Ainsworth BE, Li F. Coronavirus disease (COVID-19): The need to maintain regular physical activity while taking precautions. J Sport Health Sci. 2020;9:103-4. doi:10.1016/j.jshs.2020.02.001

12. Chawla B, Chawla S, Singh H, Jain R, Arora I. Is coronavirus lockdown taking a toll on mental health of medical students? A study using the WHOQOL-BREF questionnaire. J Family Med Prim Care. 2020;9:5261-6. doi:10.4103/jfmpc. jfmpc_715_20

13. Pagnin D, de Queiroz V. Comparison of quality of life between medical students and young general populations. Educ Health (Abingdon). 2015;28:209-12. doi:10.4103/ 1357-6283.178599

14. Solis AC, Lotufo-Neto F. Predictors of quality of life in Brazilian medical students: a systematic review and meta-analysis. Braz J Psychiatry. 2019;41:556-7. doi:10.1590/15164446-2018-0116

15. Moutinho ILD, Lucchetti ALG, Ezequiel ODS, Lucchetti G. Mental health and quality of life of Brazilian medical students: Incidence, prevalence, and associated factors within two years of follow-up. Psychiatry Res. 2019;274:306-12. doi:10.1016/j.psychres.2019.02.041

16. Souza Filho BAB, Tritany EF. COVID-19: the importance of new technologies for physical activity as a public health strategy. Cad Saude Publica. 2020;36:e00054420. doi:10.1590/0102-311x00054420

17. Stella AB, Ajčević M, Furlanis G, Cilloto T, Menichelli A, Accardo A, et al. Smart technology for physical activity and health assessment during COVID-19 lockdown. J Sports Med Phys Fitness. 2020; doi:10.23736/S00224707.20.11373-2.

18. World Health Organization. Young People's Health - A Challenge for Society. Geneva, Switzerland: World Health Organization; 1986. https://apps.who.int/iris/bitstream/handle/10665/41720/WHO_TRS_731.pdf?sequence=1\&isAllowed $=y$.

19. World Health Organization. Obesity: preventing and managing the global epidemic. Geneva, Switzerland: World Health Organization; 2000. https://www.who.int/nutrition/ publications/obesity/WHO_TRS_894/en/.

20. Fleck MPA, Louzada S, Xavier M, Chachamovich E, Vieira $\mathrm{G}$, Santos L, et al. Aplicação da versão em português do instrumento abreviado de avaliação da qualidade de vida “WHOQOL-bref". Rev Saude Publica. 2000;34:178-83. doi:10.1590/S0034-89102000000200012

21. Pardini R, Matsudo SM, Araújo T, Matsudo V, Andrade E, Braggion G, et al. Validation of the International Physical Activity Questionnaire (IPAQ-version 6): pilot study in Brazilian young adults. Rev. Bras. Ciên. Mov. 2001;9 (3):45-51.

22. Resende MA, Resende RBV, Tavares RS, Santos CRR, Barreto-Filho JAS. Estudo comparativo do perfil pró-aterosclerótico de estudantes de Medicina e de Educação Física. Arq. Bras. Cardiol. 2010;95(1):21-9.

23. Silva PGB, de Oliveira CAL, Borges MMF, Moreira DM, Alencar PNB, Avelar RL, et al. Distance learning during social seclusion by COVID-19: Improving the quality of life of undergraduate dentistry students. Eur J Dent Educ. 2020;1-11. doi:10.1111/eje.12583 
24. Xiao H, Shu W, Li M, Li Z, Tao F, Wu X, et al. Social Distancing among Medical Students during the 2019 Coronavirus Disease Pandemic in China: Disease Awareness, Anxiety Disorder, Depression, and Behavioral Activities. Int J Environ Res Public Health. 2020;17:5047. doi:10.3390/ijerph17145047

25. Alsoufi A, Alsuyihili A, Msherghi A, Elhadi A, Atiyah H, Ashini A, et al. Impact of the COVID-19 pandemic on medical education: Medical students' knowledge, attitudes, and practices regarding electronic learning. PLoS One. 2020;15:e0242905. doi:10.1371/journal.pone.0242905

26. Lira ALBC, Adamy EK, Teixeira E, da Silva FV. Nursing education: challenges and perspectives in times of the COVID-19 pandemic. Rev Bras Enferm. 2020;73: e20200683. doi:10.1590/0034-7167-2020-0683

27. Savitsky B, Findling Y, Ereli A, Hendel T. Anxiety and coping strategies among nursing students during the covid-19 pandemic. Nurse Educ Pract. 2020;46:102809. doi:10.1016/j.nepr.2020.102809

28. Lick DJ, Durso LE, Johnson KL. Minority Stress and Physical Health Among Sexual Minorities. Perspect Psychol Sci. 2013;8:521-48. doi:10.1177/1745691613497965

29. Malik VS, Ravindra K, Attri SV, Bhadada SK, Singh M. Higher body mass index is an important risk factor in COVID-19 patients: a systematic review and meta-analysis. Environ Sci Pollut Res Int. 2020; 27:42115-42123. doi:10.1007/s11356-020-10132-4

30. Peres KC, Riera R, Martimbianco ALC, Ward LS, Cunha LL. Body Mass Index and Prognosis of COVID-19 Infection. A Systematic Review. Front Endocrinol (Lausanne). 2020;11:562. doi:10.3389/fendo.2020.00562

31. Serinolli MI, Novaretti MCZ. A cross-sectional study of sociodemographic factors and their influence on the quality of life in medical students at Sao Paulo, Brazil. PLoS One. 2017;12:e180009. doi:10.1371/journal.pone.0180009

32. Romero-Blanco C, Rodríguez-Almagro J, Onieva-Zafra MD, Parra-Fernández ML, Prado-Laguna MDC, HernándezMartínez A. Physical Activity and Sedentary Lifestyle in University Students: Changes during Confinement Due to the COVID-19 Pandemic. Int J Environ Res Public Health. 2020;17:6567. doi:10.3390/ijerph17186567
33. Fröberg A. The COVID-19 pandemic: The importance of physical activity among faculty members. J Am Coll Health. 2020;1-4. doi:10.1080/07448481.2020.1817037

34. Ghram A, Briki W, Mansoor H, Al-Mohannadi AS, Lavie CJ, Chamari K. Home-based exercise can be beneficial for counteracting sedentary behavior and physical inactivity during the COVID-19 pandemic in older adults. Postgrad Med. 2020;1-12. doi:10.1080/00325481.2020.1860394

35. Silva-Filho E, Teixeira ALS, Xavier JRS, Jr Braz DS, Barbosa RA, de Albuquerque JA. Physical education role during coronavirus disease 2019 (COVID-19) pandemic. Motriz: J Phys Ed. 2020;26:e10200086. doi:10.1590/ s1980-6574202000020086

36. Tate DF, Lyons EJ, Valle CG. High-tech tools for exercise motivation: use and role of technologies such as the internet, mobile applications, social media, and video games. Diabetes Spectr. 2015;28:45-54. doi:10.2337/diaspect.28.1.45

37. Dwyer MJ, Pasini M, De Dominicis S, Righi E. Physical activity: Benefits and challenges during the COVID-19 pandemic. Scand J Med Sci Sports. 2020;30:1291-4. doi: $10.1111 /$ sms. 13710

38. Hudson GM, Sprow K. Promoting Physical Activity During the COVID-19 Pandemic: Implications for Obesity and Chronic Disease Management. J Phys Act Health. 2020;13. doi:10.1123/jpah.2020-0318

\section{Corresponding author}

José Bruno Nunes Ferreira Silva. Universidade Federal do Tocantins, Campus Universitário de Palmas, Palmas, TO, Brasil.

E-mail: nunes.brj@mail.uft.edu.br.

Manuscript received on April 26, 2021

Manuscript accepted on June 6, 2021

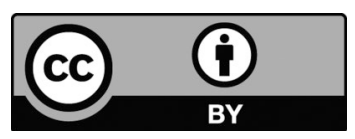

Motriz. The Journal of Physical Education. UNESP. Rio Claro, SP, Brazil - eISSN: 1980-6574 - under a license Creative Commons - Version 4.0 